\title{
14. A mile wide, inch deep: The future for Indigenous social surveys?
}

\author{
Matthew Gray
}

Shortly after the release of data from the 1994 National Aboriginal and Torres Strait Islander Survey (NATSIS), Jon Altman and John Taylor wrote: At some future time, it is likely that the undertaking of the 1994 NATSIS will be regarded as a watershed in the collection of statistics about Indigenous Australians' (Altman and Taylor 1996: 193). Some 15 years later this prediction has proven to be true. The 1994 survey and the subsequent National Aboriginal and Torres Strait Islander Social Surveys (NATSISS) conducted in 2002 and 2008 have become an important source of information on the circumstances of Indigenous Australians. However, now that we have three NATSISS spanning a decadeand-a-half, it is time to consider whether to keep repeating the NATSISS using a similar design and methodological approach, or whether a new approach is required.

The papers in this volume collectively provide an excellent summary of the strengths and limitations of the 2008 NATSISS in relation to particular topics and the types of policy-relevant questions the survey can inform. In this paper I draw together the work in the individual chapters to form an overall assessment of the strengths and limitations of the survey. The chapter also discusses some of the trade-offs that inevitably need to be made when designing a survey such as the NATSISS.

\section{Background to the development of the first national Indigenous social survey}

From 1901 until the changes to the Australian Constitution in 1967, the Constitution stated that in reckoning the numbers of the people of the Commonwealth, or of a State or other part of the Commonwealth, Aboriginal natives shall not be counted'. ${ }^{1}$ For the first Australian Census held in 1911 this section was interpreted to mean that persons of half or less Aboriginal or Torres Strait Islander descent should be included in the count of the Australian

1 Section 127 of the Commonwealth of Australia Constitution Act. 
population (Australian Bureau of Statistics (ABS) 2011). Following the 1967 Referendum, changes to the Constitution meant that Indigenous people were to be counted in the census. This took effect in the 1971 Census and so we have census data covering all Indigenous people from the 1971 Census onwards.

This has meant that since 1971 there has been relatively comprehensive national data on the number and demographic characteristics of Indigenous Australians and some data on the economic and social circumstances. The censuses do not cover many important issues including physical and mental health, wellbeing or subjective data on experiences. In other areas the censuses provide only limited data (e.g. labour market, housing circumstances). There has been some expansion in the range of areas the census provides data with the 2006 and 2011 Censuses including questions about the need for assistance with daily living, care provided to people with a disability and voluntary work.

Until the 1994 NATSIS the Census remained virtually the only broad based national data on Indigenous Australians. The 1994 NATSIS in fact arose from a recommendation of the Royal Commission into Aboriginal Deaths in Custody that there be 'a special national survey covering a range of social, demographic, health and economic characteristics of the Aboriginal population with full Aboriginal participation' in order to fill an information void on the interrelationships between different aspects of Indigenous disadvantage (Commonwealth of Australia 1991). The Royal Commission also recommended that there be improved indigenous identification on administrative data bases. ${ }^{2}$

Both of these data related recommendations of the Royal Commission have been acted upon. The Australian government provided additional funding to the ABS to undertake a national social survey of the Indigenous population. There has also been a very substantial increase in the amount of administrative data which identifies Indigenous individuals and can therefore be used to provide information on their circumstances and experiences (e.g. ABS 2007).

The extent of the increase in the amount of data available about Indigenous Australians circumstances is illustrated by the Productivity Commission's regular report Overcoming Indigenous Disadvantage Report which was first published in 2003 (Steering Committee for the Review of Government Service Provision (SCRGSP) 2003). Between the 2003 and 2011 reports there has been a significant improvement in the data that could be used by the Productivity Commission to measure change in Indigenous outcome across a wide range of wellbeing measures.

While the range and quality of data available has improved, there is a heavy reliance on data from administrative sources. For example, the 2011 Overcoming

2 Recommendations 49 and 68 (Commonwealth of Australia 1991). 
Indigenous Disadvantage Report uses data from around 25 administrative sources, ${ }^{3}$ but uses data from only a handful of social or health surveys. In addition to data from the population censuses, there are just two nationally representative surveys used by the Productivity Commission: the NATSISS series of surveys (1994, 2002 and 2008) and the two most recent National Health Surveys that include an augmented sample of Indigenous Australians. ${ }^{4}$ The NATSISS remains the main source of survey data on a wide range of aspects of the lives of Indigenous Australians. ${ }^{5}$

In many respects the administrative and survey collections are complementary sources of data with the administrative data often mainly providing information on service use, something that is difficult to collect in surveys. However, the administrative data generally does not include a range of other variables (such as educational attainment) which are central to understanding behaviour and the reasons for differences in outcomes.

\section{Strengths and limitations of the 2008 NATSISS}

The overall conclusion which emerges from the chapters in this book is that while the NATSISS provides information on a wide range of topics, for most topics the data collected is high level and that for some topics, the survey provides only superficial information. This limits the utility of the survey for addressing key policy relevant questions. It is a survey that is a mile wide and an inch deep.

In some chapters, the authors conclude that the data is very useful for addressing important policy questions and the authors put the data to good effect in demonstrating how it can be used to help inform such questions. In other chapters in this book the authors conclude that the 2008 NATSISS data has only limited utility. Examples of such areas are alcohol use (Chikritzhs and Liang), the customary economy (Altman, Biddle and Buchanan), demography and particularly fertility (Johnstone and Evans), ${ }^{6}$ geographic mobility (Taylor and Bell) and housing (Memmott et al.). Biddle and Cameron find the education

\footnotetext{
3 For the purposes of this calculation, large-scale monitoring surveys such as the Australian Early Development Index and the Australian Government Department of Education, Employment and Workplace Relations post-program monitoring surveys are classified as administrative sources.

4 In addition, two more narrowly focused dental health surveys are used.

5 Longitudinal data has become increasingly important in the social sciences. There is little longitudinal data available on Indigenous Australians, but an important recent development has been the Longitudinal Study of Indigenous Children (LSIC) being run by the Department of Families, Housing, Community Services and Indigenous Affairs.

6 While the ABS publication Births Australia provides data on fertility, this data set does not provide the range of variables required to understanding the determinants and outcomes of fertility (ABS 2010).
} 
data so limiting that they in fact base much of their chapter on analysis of data from the Longitudinal Survey of Australian Youth which oversamples Indigenous young people and thus provides a substantial Indigenous sample.

While the breadth is a weakness it also a strength. Omnibus surveys such as the 2008 NATSISS can be used to analyse complex interrelationships between economic, social and cultural factors, something which generally cannot be done using administrative data or more narrowly focused surveys that arguably 'silo' the different aspects of human experience. It is also a framework against which more detailed studies and surveys can be developed in order to provide a great depth of understanding about particular topics. While there is a strong case for breadth in a survey such as the NATSISS, in order to be able to examine complex interrelationships the data on each topic needs to have enough depth to allow a convincing analysis.

The 2008 NATSISS includes a number of new modules. The main ones are: the inclusion of children aged 0-14 years in the sample (information about the children provided by an adult, usually a parent or other relative who has caring responsibility for the child); experience of discrimination; and broadening of the measures of wellbeing to include mental health and subjective wellbeing. While these topics are extremely important and are areas in which there is a real shortage of quantitative information, the space in the questionnaire for their inclusion has been mostly created by reducing the depth of questions in other areas, rather than by dropping entire areas of questions. This has exacerbated the problem of the survey being broad but shallow.

\section{Key policy relevant research questions}

There are many policy relevant questions which social survey data could help address, but for which the necessary survey data is not available. Some examples or questions on which the NATSISS 2008 provides data include:

- Does the maintenance of traditional cultural beliefs and practices act as a barrier to achieving outcomes such as subjective wellbeing in health, educational attainment, employment and financial wellbeing or does it contribute to higher levels of wellbeing in such areas. ${ }^{7}$

- Does migration to areas with better educational and employment opportunities result in increased rates of educational participation and employment? Does moving to larger towns or cities have an impact upon a range of other dimensions of wellbeing? Are there some areas of wellbeing where there is a

7 This issue has been the subject of considerable debate. See for example, Dockery (2010) and Hughes (2007). 
positive effect and others in which this pattern of geographic mobility has a negative effect? The answer to these questions has implications for the extent to which government policies should actively encourage migration to areas with greater educational and economic opportunities and better services.

- What are the impacts of mobility on the demography of the population in different areas and on service delivery needs?

- What are the factors that contribute to some Indigenous children doing well and others not so well? What promotes resilience amongst Indigenous children and what places them at risk?

- To what extent do Indigenous Australians experience discrimination, in what contexts is this most likely to occur and what impact does the experience of discrimination have on different aspects of individual lives?

- To what extent do Indigenous communities benefit from mining activities that occur near their community and in some cases on their land?

- What are the predictors of Indigenous fertility and how is this likely to change in the future?

A number of the chapters in this volume conclude that due to the necessary depth of information not being collected (or in some cases not collected at all), the 2008 NATSISS is of only limited use in answering these questions. This point is illustrated by questions about the impacts of maintaining traditional cultural beliefs and practices. The 2008 NATSISS data is used in chapters in this volume to explore the links between cultural beliefs and practices and other aspects of Indigenous peoples' lives. The chapter by Dockery uses this data to test the relationship between stronger cultural attachment and participation in cultural activities (at least as measured in the 2008 NATSISS) and Weatherburn and Snowball in their chapter use the data to test whether there is support for cultural theories of Indigenous violence. Both of these papers find no evidence that traditional cultural practices and beliefs are associated with worse outcomes in these two dimensions. Ultimately these types of questions can probably only be adequately addressed using mixed methods approach and triangulation of data from different sources.

While the findings of Dockery, and Weatherburn and Snowball are important, there remains a question mark as to whether the 2008 NATSISS items capture cultural practices and beliefs that are sometimes argued to be problematic to operating in a modern market based economy. It is fair to say that the 2008 NATSISS questionnaire only collects data on 'positive' aspects of culture (or the absence of participation in these aspects of culture).

An example of the type of cultural practice that is sometimes considered problematic is traditional demand-sharing practices which it is argued can reduce the incentives of individuals to look for and accept paid employment 
(Austin-Broos 2006; Musharbash 2001). This is not an issue on which the NATSISS 2008 provides data and it is arguable as to whether it is possible to meaningful collect this type of information in a large-scale quantitative survey, particularly one conducted by the Australian Government's official statistical agency. The lack of data on the potentially negative aspects of culture means that the conclusions about the links between traditional cultural practices and beliefs need to be treated with caution.

In relation to geographic mobility, Taylor and Bell in their chapter conclude that while the survey is useful for understanding the nature of mobility, it is limited in its ability to inform discussions about the relationship between policy and population movement. For example, the way in which the 2008 NATSISS data have been coded for public release means that it is not possible to get a reliable measure of whether people are moving from smaller to larger places or whether they are moving from larger to smaller places.

The NATSISS data is provided to researchers in a confidentialised form. As part of this process the data is aggregated into broad geographic areas. An almost constant refrain in the chapters in this book is that the geographic classification used by the ABS is too broad and this severely limits the extent to which the data can be used to provide estimates according to geographic remoteness. This is a pity because the relatively large sample size of NATSISS opens up the possibility of using the data to understand the extent to which government policies are likely to have differential impacts in different parts of the country. There are many other questions which the NATSISS data could potentially answer, if more detailed geographic information were to be released. Just one example is the question of whether living near a significant mine has an impact upon the economic and social well being of individuals.

While it is essential that the data be confidentialised in such a way that release of the data is not likely to lead to identification of the persons or organisations to which it relates, this can be achieved in a variety of ways and with differing thresholds of likelihood of identification of individuals. The ABS has taken an approach which results in a greater level of geographic aggregation than is the case for some other government funded surveys such as the Household, Income and Labour Dynamics in Australia (HILDA) survey and the Longitudinal Study of Australian Children (LSAC).

One of the objectives of the NATSISS surveys is to measure change for the Indigenous population. While this is one of the strengths of these surveys, several of the chapters point to changes that have been made to the questionnaire between surveys that mean that this is not possible for certain topics. For example, Altman, Biddle and Buchanan argue that in the area of participation in the customary sector, modifications to the questionnaire mean that change 
between 2002 and 2008 can't be estimated. Similarly, Taylor and Bell argue that variations to the questionnaire means that changes in mobility between NATSISS collections can't be estimated. In contrast, the chapter on the NATSISS labour force status data (Thapa, Shah and Ahmad) provides a good illustration of how labour force status is changing.

In designing the next wave of NATSISS, emphasis should be placed on maintaining comparability of measures with earlier NATSISS undertaken. As the Productivity Commission's 2011 Overcoming Indigenous Disadvantage Report finds, despite the increases in the amount of data available about Indigenous Australians, it is hard to know how things are changing for Indigenous Australians and to assess whether current policy approaches are working and what changes in approach may be needed (SCRGSP 2011). This emphasises the importance of future social surveys of Indigenous Australians maintaining a high degree of comparability with the 2008 survey.

\section{Objective versus subjective measures of wellbeing}

The inclusion of both objective and subjective measures of wellbeing in the 2008 NATSISS highlights a very important issue. According to virtually any objective measure of wellbeing (at least those identified in the Closing the Gap policy agenda), Indigenous Australians have poorer outcomes than are found in the Australian population as a whole. Yet, on many of the subjective assessments of wellbeing Indigenous Australians rate their wellbeing quite highly.

Some examples of this are provided by the chapters in this volume. According to parents, rating of the overall health status of their child, only 1 in 25 children had fair or poor health with the majority in excellent or very good health. Furthermore, according to parental assessment, the health of children in remote and non-remote areas was similar (Shepherd and Zubrick). This assessment of child health differs to more objective measures of health. For example, Indigenous women are twice as likely as non-Indigenous women to have a low birth weight baby and Indigenous children are more likely to have been hospitalised than are non-Indigenous children (SCRGSP 2011).

While the data on children is a valuable addition to the survey, the question of the reliability of the child's carer (often a parent) to assess children's developmental outcome needs further examination. Shepherd and Zubrick find that the majority of Indigenous children are in excellent or very good overall health, although there are some developmental danger signs for a significant number of children. Other evidence suggests that Indigenous children are more 
likely than other children to have significant health problems (e.g. Zubrick et al. 2004). Further research is required on how parental assessment of the health of Indigenous and non-Indigenous children compare, and how the assessments by the parents of Indigenous children compare to the picture that would emerge if clinical assessment rather than parental self-report was used. An important question is whether the higher rates of health problems and poorer developmental outcomes found amongst Indigenous children in some communities means that parental perceptions of what it means for a child to be healthy or to have normal developmental outcomes is lowered by the poor outcomes in their community.

Another example relates to children's happiness at school. Indigenous Australians are on average happier at school at the age of 15 than non-Indigenous Australians (data from the Longitudinal Survey of Australian Youth). ${ }^{8}$ The objective outcome is that Indigenous children are less likely to be at school at the age of 15 than other Australian children and have far poorer academic outcomes (SCRGSP 2011).

Self-reported health status provides a slightly different example of this general issue. While Indigenous Australians self-reported health status is lower overall than that of other Australians, Indigenous Australians in remote areas report having better health than those in non-remote areas. This finding is surprising given that most clinical data suggests the reverse. ${ }^{9}$ In contrast, the more objective measures of wellbeing (or at least socioeconomic outcomes) such as income, experience of financial hardships, paid employment, and education collected in the NATSISS 2008 are much lower for the Indigenous population that is found for the Australian population as a whole.

It is hard to know how to interpret these data, although there are a range of possible explanations. One is that subjective assessments of wellbeing are usually based on a social comparison and are thus relative. The social norms against which many Indigenous people assess their circumstances may differ to that used by the overall Australian population and therefore Indigenous people may give a higher assessment of their subjective wellbeing than a nonIndigenous person in the same objective circumstances. There may be adaptation and habituation to continuing conditions of disadvantage and poor health. Third, the answers to the survey questions may be affected by the respondent's desire to not look bad to the interviewer; to not 'be shamed' (social desirability bias). Another possible explanation is that doing well in areas such as paid

8 Further information on the Longitudinal Survey of Australian Youth is available from the study website $<$ http://www.lsay.edu.au>

9 This issue was discussed in Cunningham, Sibthorpe and Anderson (1997) based on their analysis of the 1994 NATSIS. 
employment, income, education and housing circumstances are less important for the subjective wellbeing of Indigenous people than is the case for nonIndigenous Australians. ${ }^{10}$

Clearly, there are at least some differences between Indigenous and nonIndigenous people in what is important in determining their wellbeing (e.g. Dodson 2012). As Taylor (2008: 123) writes 'Indigenous peoples' perceptions and understandings of well-being extend beyond, and sometimes conflict with, many of the indicators currently adopted by global reporting frameworks.' and could include factors such as ability to access ritual or religious knowledge and reciprocity in social and economic relations.

It is not possible using the 2008 NATSISS data to assess the extent to which the disparity between subjective and objective measures of wellbeing for Indigenous Australians is explained by difference in social norms, a process of habituation or adaptation, or qualitatively distinct determinants of wellbeing between Indigenous and non-Indigenous. However, resolving this question does have important implications for both assessing the current circumstances of Indigenous Australians and policies specifically aimed at improving the wellbeing of this group. The answer to this question has implications for the extent to which closing the gap in outcomes between Indigenous and nonIndigenous Australians should be a measure of policy success, and whether it is sensible to use closing gaps in some - but not all - of the outcome measures which currently are the target of policy.

\section{What should the future be for national social surveys of Indigenous Australians?}

This question needs to be considered in the context of other data developments, particularly in relation to the recent improvements in administrative data. While such data is very useful, its main limitation is that the range of data collected is generally restricted to that required to administer a program. This means that it generally provides information on only a limited range of factors and cannot be used to examine inter-relationships between outcomes and more complex causal pathways. $^{11}$

10 An overview of the research on subjective wellbeing is provided by Deiner et al. (1999).

11 While, in principal, this limitation can be at least partially overcome by linking different sources of data, in practice linking administrative data sets is very difficult, not always technically possible, and can be subject to privacy related-concerns. Nonetheless there are a range of data linkages currently in existence or in the development phase including the ABS Indigenous Identification Mortality Data Linkage Project. 
The other major limitation of administrative data is that it does not provide information on Indigenous people's own assessments of their circumstances and relationships (subjective wellbeing measures) and what is important to them. There are ongoing concerns in relation to accurate identification of Indigenous people in administrative datasets although this has been much improved recently. Of course administrative data have strengths as well, including that they do not increase the burden on respondents; that they usually provide accurate information on individual's interactions with government or government funded services; that they often provide objective measures of people's circumstances (e.g. health services used); and the collection often provides longitudinal data (although this can be limited by privacy concerns).

\section{Indigenous involvement in and control over social surveys of Indigenous Australians}

The difficulty of collecting data on Indigenous cultural practices and beliefs partly arises from data collection by Australia's national statistics office. The opening paper at the Social Science Perspectives on the 2008 National and Aboriginal Torres Strait Islander Social Survey conference by Peter Yu (delivered by his Yawuru countryman, Professor Mick Dodson) was about the power of data in Aboriginal hands. Yu (2011: 1-2) writes:

... I want to talk about the critical role that data can play in development scenarios, when Aboriginal people are in control of collecting, managing and interpreting data... The view I have about data is a long way from the current paradigm where data is collected on Indigenous society by governments for their purposes; rather than to support the objectives that Indigenous people want to determine.

... However, one has to look only as far as the National Aboriginal Torres Strait Islander Social Survey conducted by the ABS to appreciate how far this reform agenda has to travel. That ABS survey is designed to assist governments, commentators or academics who want to construct policies that shape our lives or encourage a one sided public discourse about us and our position in the Australian nation.

The view presented by Yu is a fundamental challenge to the ABS and the largely non-Indigenous users of the NATSISS data. On the one hand it is important that data is collected which allows the circumstance of Indigenous Australians to be understood through the lens of non-Indigenous Australian culture. On the other it is vital that Indigenous people are increasingly involved in collecting, managing and interpreting the data collected pertaining to them and their communities. 


\section{Looking ahead}

All surveys involve trade-offs, and any resulting survey design has tensions within. Trade-offs are required for a range of reasons including budget and respondent burden. The NATSISS is no different and, perhaps because of its position as THE national social and economic survey of Indigenous Australians, it faces more constraints than many other surveys.

This chapter has argued that the 2008 NATSISS has erred on the side of being too broad and lacking depth. This outcome is perhaps not surprising given that the NATSISS is the only large-scale nationally representative social survey of Indigenous Australians. ${ }^{12}$

In considering the future design of social surveys of Indigenous Australians, it is timely to review the objectives of the 1994 NATSISS and their relevance to twenty-first century needs. The objectives of the 1994 NATSISS were to 'provide the most needed statistics in a range of social, demographic, health and economic areas, thereby providing a stronger information base for planning for the empowerment of Australia's Indigenous people and for measuring progress in meeting their objectives, aspirations and needs' (Sarossy 1996: 190). While these objectives are laudable and remain relevant, it is worth reconsidering what and who should be the primary purpose and the primary users of the survey.

The range of potential objectives for social surveys of Indigenous Australians, such as the NATSISS, include:

- monitoring progress in closing gaps in outcomes between Indigenous and non-Indigenous Australians and in changes in the circumstances of Indigenous peoples over time

- providing information on the distinctive nature of the lives of Indigenous Australians, their aspirations and their experiences, and

- empowering Indigenous communities by providing information which they can use to plan and advocate on behalf of members of their communities, in order to improve their lives.

In my view, it is not possible for a social survey of Indigenous Australians to simultaneously meet all three purposes. The future approach depends upon which of these objectives is given priority. If it is monitoring progress in closing gaps between Indigenous and non-Indigenous Australians, then there needs to be a high priority placed on maximising the comparability between the questions in the Indigenous-specific social survey and question in other surveys of the general Australian population. If this is the key objective of the survey

12 The Indigenous health surveys run by the ABS are focused on health, and therefore provide limited information on other areas of individual lives. 
then it may be worth considering supplementing other survey samples (such as the Labour Force Survey) with a sample of Indigenous Australians, rather than using an Indigenous-specific social survey. A further implication is that there needs to be no or minimal change in the variables analysed. This position is becoming more defensible as the range of administrative sources of data that identify Indigenous Australians increases and the prospects for carefully considered data linkage across administrative sources improve.

If the main aim is to provide information on the distinctive nature of the lives of Indigenous Australians, then the survey will have less overlap with other existing surveys and there clearly needs to be a separate Indigenous specific social survey. This approach would mean that the content of NATSISS would have much less overlap with those of other surveys of the general Australian population run by the ABS. The greater the focus is on the distinctive nature of the lives of Indigenous Australians the more important becomes Indigenous involvement in the design and conduct of the survey.

If priority is given to the third objective, then this would require a total rethink of the NATSISS. Meeting such an objective would probably require communitylevel surveys, and the control over the content and nature of data collection would be quite different. Surveys designed to meet such an objective would be driven by Indigenous communities and likely result in surveys quite different to anything undertaken up to the present. Under these conditions, a national survey such as the NATSISS would remain essential in order to gain an understanding of the circumstances of Indigenous people in different communities and to allow a national picture to be painted. There are some initial steps being taken in this area with some communities undertaking such surveys, including in Broome (Taylor et al. 2012; Yu 2011).

A social survey such as the NATSISS can ultimately never tell those responsible for developing public policy what to do, but it can provide useful information to inform policy decisions. The chapters in this monograph cover a wide range of topics and illustrate ways in which NATSISS 2008 data can be used, and the strengths and weaknesses of the data. Taken as whole, they demonstrate that NATSISS 2008 can contribute to our understanding of the diversity of circumstances in which Indigenous Australians live. They also demonstrate that for many areas the NATSISS has become too broad and too shallow to be useful in informing policy making. It is time to rethink the real purpose of social surveys of the Indigenous population and to focus the design of the survey on this purpose. 


\section{Acknowledgements}

The author is grateful to Kim Farley-Larmour, Boyd Hunter, David Stanton, John Taylor and two anonymous referees for comments on an earlier version of this chapter.

\section{References}

Altman, J. and Taylor, J. 1996. 'Statistical needs in Indigenous affairs: future options and implications', in J. Altman and J. Taylor (eds), The 1994 National Aboriginal and Torres Strait Islander Survey: Findings and Future Prospects, CAEPR Research Monograph No. 11, CAEPR, ANU, Canberra.

Austin-Broos, D. 2006. "Working for" and "working: among Western Arrernte in Central Australia', Oceania, 76 (1): 1-15.

Australian Bureau of Statistics (ABS) 2007. Directions in Aboriginal and Torres Strait Islander Statistics, June 2007, cat. no. 4700.0, ABS, Canberra.

2010. Births Australia 2009, cat. no. 3301.0, ABS, Canberra.

2011. Reflecting a Nation: Stories from the 2011 Census, cat. no. 2071.0, ABS, Canberra.

Carson, B., Dunbar, T., Chenhall, R. and Bailie, R. (eds) 2007. Social Determinants of Indigenous Health, Allen \& Unwin, Crows Nest.

Cunningham, J., Sibthorpe, B. and Anderson, I. 1997. Occasional Paper: SelfAssessed Health Status, Indigenous Australians, cat. no. 4707.0, ABS, Canberra.

Diener, E., Eunkook, M., Suh, R. and Smith, H. 1999' 'Subjective well-being: Three decades of progress', Pyschological Bulletin, 125 (2): 276-302.

Dodson, P. 2012. Mahatma Gandhi Inaugural Oration, 30 January 2012, University of New South Wales, Sydney.

Dockery, A. M. 2010. 'Culture and wellbeing: The case of Indigenous Australians', Social Indicators Research, 99 (2), 315-32, DOI: 10.1007/s11205-010-9582-y.

Hughes, H. 2007. Lands of Shame: Aboriginal and Torres Strait Islander 'Homelands' in Transition, Centre for Independent Studies, Sydney. 
Musharbash, Y. 2001. 'Yuendumu CDEP: The Warlpiri work ethic and Kardiya staff turnover', in F. Morphy and W. Sanders (eds), The Indigenous Welfare Economy and the CDEP Scheme, CAEPR Research Monograph No. 20, ANU E Press, Canberra.

Commonwealth of Australia 1991. Royal Commission into Aboriginal Deaths in Custody, National Report, Vol. 5, AGPS, Canberra.

Steering Committee for the Review of Government Service Provision (SCRGSP) 2003. Overcoming Indigenous Disadvantage: Key Indicators 2003, Productivity Commission, Canberra.

- 2011. Overcoming Indigenous Disadvantage: Key Indicators 2011, Productivity Commission, Canberra.

Sarossy, G. '1996. 'Findings from the NATSIS evaluation', in J. Altman and J. Taylor (eds), The 1994 National Aboriginal and Torres Strait Islander Survey: Findings and Future Prospects, CAEPR Research Monograph No. 11, CAEPR, ANU, Canberra.

Taylor, J. 2008. 'Indigenous people and indicators of well-being: Australian perspectives on United Nations global frameworks', Social Indicators Research, 87: 111-26.

—, Doran, B., Parriman, M. and Yu, E. 2012. 'Statistics for community governance: The Yawuru Indigenous population survey of Broome', CAEPR Working Paper No. 82, CAEPR, ANU, Canberra.

Yu, P. 2011. 'The power of data in Aboriginal hands', Paper read by Professor M. Dodson, Social Science Perspectives on the 2008 National and Aboriginal Torres Strait Islander Social Survey Conference, 11-12 April, CAEPR, ANU, Canberra, available at <http://caepr.anu.edu.au/seminars/conferences/ natsis2011>

Zubrick, S., Lawrence, D., Silburn, S., Blair, E., Milroy, H., Wilkes, T., Eades, S., D'Antoine, H., Read, A., Ishiguchi, P. and Doyle, S. 2004. The Western Australian Aboriginal Child Health Survey: The Health of Aboriginal Children and Young People, Telethon Institute for Child Health Research, Perth. 